\title{
BMJ Open Assessing vaccine hesitancy in Arab countries in the Middle East and North Africa (MENA) region: a scoping review protocol
}

\author{
Bayan Galal (D , ${ }^{1}$ Stefany Lazieh, ${ }^{2}$ Samir Al-Ali, ${ }^{1}$ Kaveh Khoshnood ${ }^{3}$
}

To cite: Galal B, Lazieh S, AlAli $\mathrm{S}$, et al. Assessing vaccine hesitancy in Arab countries in the Middle East and North Africa (MENA) region: a scoping review protocol. BMJ Open 2022;12:e045348. doi:10.1136/ bmjopen-2020-045348

- Prepublication history for this paper is available online. To view these files, please visit the journal online (http://dx.doi. org/10.1136/bmjopen-2020045348).

Received 28 September 2020 Accepted 14 July 2021

Check for updates

(c) Author(s) (or their employer(s)) 2022. Re-use permitted under CC BY-NC. No commercial re-use. See rights and permissions. Published by BMJ.

${ }^{1}$ Yale University, New Haven, Connecticut, USA

${ }^{2}$ Rutgers University School of Arts and Sciences, New Brunswick, New Jersey, USA ${ }^{3}$ Department of Epidemiology of Microbial Diseases, Yale University, New Haven, Connecticut, USA

Correspondence to

Bayan Galal;

bayan.galal@yale.edu

\section{ABSTRACT}

Introduction Vaccine-preventable disease outbreaks have increased in past years, and there is great public health interest in monitoring attitudes towards vaccination as well as identifying factors contributing to vaccine hesitancy and refusal. Although the WHO declared vaccine hesitancy as one of the top threats to global health in 2019, studies focused on the determinants and extent of vaccine hesitancy in Arab countries in the Middle East and North Africa (MENA) region are lacking. This scoping review explores the various factors surrounding vaccine hesitancy, including but not limited to geographic, cultural and religious factors, and examines the extent and nature of the existing evidence on this topic. In light of current development of various COVID-19 vaccines, our work seeks to elucidate the barriers to vaccine uptake in specific populations.

Methods and analysis This review will be conducted using the Joanna Briggs Institute Manual for Scoping Reviews. It will comply with the Preferred Reporting Items for Systematic Reviews and Meta-Analyses extension for Scoping Reviews guidelines. Studies published in English, Arabic and French between January 1998 and December 2020 will be drawn from PubMed, Embase, Cochrane and Scopus. The search strategy will include terms related to vaccination and vaccine hesitancy in Arab countries in the MENA region. We will also include grey literature on the topic by searching Google and Google Scholar. Studies will be selected according to the ParticipantsIntervention-Comparators-Outcome model, and all study titles and abstracts will be screened by two reviewers. Disagreements will be resolved with a third reviewer's input.

Ethics and dissemination This review is exempted from ethical approval and will be published in a peer-reviewed open-access journal to ensure wide dissemination.

\section{INTRODUCTION}

According to a 2019 report from the WHO, one of the 10 threats to global health is vaccine hesitancy, which is defined as the reluctance or refusal to vaccinate despite the availability of vaccines. ${ }^{12}$ Vaccination is internationally recognised as one of the most successful public health measures, currently preventing 2-3 million deaths each year, according to the
Strengths and limitations of this study

- This review will integrate evidence of vaccine hesitancy in Arab countries in the Middle East and North Africa region.

- This review will include all types of evidence sources published in English, Arabic and French.

- The literature search will comply with the Preferred Reporting Items for Systematic Reviews and MetaAnalyses extension for Scoping Reviews checklist.

- One possible limitation of this review will be the exclusion of a meta-analysis for this scoping review.

WHO. Global vaccination efforts are credited with eradicating smallpox and controlling a number of infectious diseases worldwide, including measles, rubella, tetanus, diphtheria, Haemophilus influenzae type $\mathrm{b}$ and many others. ${ }^{3}$

Vaccine success relies heavily on herd immunity, which is a population's resistance to the spread of a contagious disease if a sufficiently high proportion of individuals are immune to the disease. ${ }^{4}$ This principle is exemplified most recently by the COVID-19 pandemic where social distancing and quarantine measures have been implemented to limit interactions and prevent spread of the virus. Countries who abide by public health guidelines have seen greater success in containing the virus and are better prepared to implement a COVID-19 vaccine programme if one is developed in the future.

A measles outbreak in France in $2010^{5}$ and one of the largest measles epidemics in North America in $2011^{6}$ highlighted the importance of achieving sufficient vaccine coverage as well as fully completing vaccination series for effective resistance. Despite the well-documented benefits of universal vaccinations, a growing portion of the population is perceiving vaccinations as unsafe or unnecessary and are thus avoiding or refusing 
vaccinations for their children, especially in developed countries. $^{7}$

In order to avoid devastating infectious disease outbreaks in developing countries and across the world, it is crucial to work towards complete and comprehensive immunisation coverage. In Arab countries in the Middle East and North Africa (MENA) region, there have been few studies examining determinants associated with vaccine refusal and hesitancy. This scoping review aims to identify and summarise the factors and causes of vaccine hesitancy among Arab populations in the MENA region by examining the extent of the existing evidence on the subject.

\section{MENA-specific issues}

The MENA region is not homogenous in culture or religion, and while we are interested in exploring the geography-specific factors that contribute to vaccine hesitancy, we also aim to explore the cultural and religious factors characteristic of Arab countries. Thus, this proposed review will focus on Arab countries in the MENA region, which, according to the World Bank, includes Algeria, Bahrain, Djibouti, Egypt, Iraq, Jordan, Kuwait, Lebanon, Libya, Morocco, Oman, Qatar, Saudi Arabia, Syria, Tunisia, the United Arabic Emirates, West Bank and Gaza, and Yemen. ${ }^{89}$

While there are limited studies into the factors contributing to vaccine hesitancy in these countries, there have been some studies investigating childhood vaccine refusal. A cross-sectional study in Saudi Arabia found that in a population of 500 parents, $20 \%$ were hesitant to get their child vaccinated. Parents with higher educational levels were more vaccine hesitant, and concerns related to vaccine safety were cited by most vaccine-hesitant parents. ${ }^{10}$ This relationship between education level and vaccine hesitancy merits further study to perhaps identify region-specific factors that complicate vaccine acceptance. These studies highlight the importance of tailoring provaccination efforts in the region to address the main concerns and unique trends of the area.

Most of the Arab countries in the MENA region are Muslim-majority countries, so in addition to identifying general sentiments against vaccinations, studying Muslimspecific factors could provide greater insight into the social and religious determinants of vaccine hesitancy in this area. ${ }^{11}$ While the opposition to vaccines can be traced back as far as the 18 th century, ${ }^{11}$ recent surges in antivaccination sentiments have manifested as social movements in the Western world and on social media. Some social media posts have even spread misinformation among Middle Eastern and Muslim populations by claiming that the vaccine is a plot to weaken Muslims or transmit diseases to non-Western communities. ${ }^{12}$ Another pressing issue is the need for halal-certified vaccines. Islamic law mandates that Muslims must not use any medicine or ingredients from haram sources (ingredients containing pig or its derivatives). When the 'halal' status of a vaccine is doubted, parents often turn to alternative or homeopathic medicines; this may be supported by findings from Zuzak et al, whose study reported lower vaccination rates among complementary medicine users. ${ }^{13}$ Efforts to ensure that vaccines are manufactured to meet halal requirements, if scientifically possible, and that vaccines are clearly labelled as halal certified could convince Muslim communities and individuals of the safety surrounding vaccines. While health authorities in Pakistan and Malaysia, for example, have imposed punishments on parents refusing vaccines, ${ }^{14}$ the MENA region could benefit from campaigns that specifically address religious concerns in order to ensure higher acceptance rates.

Hesitancy or refusal towards specific vaccines has also been noted in the MENA region. Several Muslim-majority countries have seen a spike in diseases preventable by vaccination, including measles and influenza. In 2009, Saudi Arabia saw more than $80 \%$ of parents refuse to authorise vaccination of their children against influenza, while Egypt recorded nearly 5000 children infected with measles in $2015 .^{15}$

Countries in the MENA region have also contended with human papillomavirus (HPV) and varying degrees of vaccination against the virus. Several of these countries have documented a notable incidence of cervical and other HPV-related cancers which are effectively prevented against with the HPV vaccine. A 2013 study by Vaccarella et al indicated that while cervical cancer is the fourth most common cancer in the larger MENA region, it exists among women in Algeria and Morocco as the second most common cancer and the third most common cancer among those in Tunisia, Oman and the United Arab Emirates (UAE). ${ }^{15}$ In September 2018, the UAE Ministry of Health and Prevention announced that all female students from eighth grade onwards would be required to be vaccinated against HPV or cervical cancer. This policy was met with public backlash and resulted in the ministry altering its policy to require parent approval before vaccination. ${ }^{16}$ A 2018 systematic review of literature on HPV vaccine acceptance in the MENA region indicated that factors influencing acceptability of the HPV vaccine often included concerns about its safety, which the study suggests would be mitigated by public sensitisation to HPV and awareness campaigns providing assurance of the vaccine's effectiveness. ${ }^{17}$

\section{Effects of COVID-19}

Despite the importance of vaccinations, immunisation schedules can be interrupted or postponed due to global events. Currently faced with the COVID-19 pandemic, more than half of the world's population was forced into lockdown to limit the spread of the virus. While the effects of COVID-19 have undoubtedly permeated all facets of society, there has been an especially notable decrease in vaccinations for other diseases due to suspended healthcare resources, fears of contracting the virus and mistrust in healthcare systems. ${ }^{18}$ Fears of infectious disease outbreaks due to disruptions of children's vaccination 
schedules must be factored into reopening procedures across the world. Furthermore, COVID-19 vaccine hesitancy may prove to be a formidable barrier to the ongoing COVID-19 epidemic: 10 days after France established a nationwide lockdown, a survey of French adults revealed that if a vaccine against SARS-CoV-2 became available, $26 \%$ of people would refuse it. ${ }^{19}$ Socioeconomic status, gender, age and even political views all factor into individuals' views on receiving vaccines, so re-establishing regular vaccine schedules after COVID-19 will depend on more than availability and accessibility.

In the MENA region, the aforementioned factors which already complicate vaccine acceptance will only be amplified amid the pandemic. Identifying and tailoring resources to decrease vaccine hesitancy in this region before the development of a COVID-19 vaccine will be crucial in order to achieve and maintain higher vaccine acceptance in the region.

Vaccine hesitancy has, of late, become an extremely pressing public health issue, and in anticipation of the development of a COVID-19 vaccine possibly within the next year, addressing vaccine uptake is essential for complete suppression of the coronavirus pandemic. Given the dearth of vaccine hesitancy studies in the MENA population, this proposed scoping review will be useful for identifying gaps in this research area. ${ }^{20}$ Though our review focuses on vaccine hesitancy, we recognise that this is not the sole factor for underimmunisation in Arab populations. Through this proposed review, we may also highlight other factors contributing to lower immunisation levels in these populations, but we do believe that identifying factors contributing to vaccine hesitancy specifically can better prepare us to identify emerging concerns before they evolve into vaccine confidence crises'. ${ }^{21}$ Thus, the main objectives for this scoping review are as follows: (1) identify evidence surrounding the causes of vaccine hesitancy among Arab populations in the MENA, (2) examine the extent and nature of the existing evidence, and (3) determine gaps in existing research surrounding this topic. Given these objectives, our main question is 'What are the main factors contributing to vaccine hesitancy among Arab populations in the Middle East and North Africa?'

\section{METHODS AND ANALYSIS}

The methodological framework for the scoping review will be based on the Joanna Briggs Institute (JBI) Manual for Scoping Reviews, ${ }^{22}$ and it will comply with the Preferred Reporting Items for Systematic Reviews and Meta-Analyses extension for Scoping Reviews guidelines. The manual outlines the following components: title, background, review question/objective, inclusion criteria, types of participants, concept, context, searching, extracting and charting the results, discussion, and conclusions and implications for research and practice. Each of these components will be addressed in the methods and analysis section.

\section{Inclusion criteria}

Articles will be included if they focus on the theme of vaccine hesitancy and its variations (including but not limited to vaccine acceptance, vaccine confidence, vaccine attitudes and behaviours, trust, distrust, concerns, perceptions and beliefs about vaccines and vaccination programmes), with a particular focus on an Arab country or countries in the MENA region. Given the already limited scope of literature present on the topics, the articles will not need to have a specific comparator but, instead, simply need to assess vaccine hesitancy and its variation in the listed countries.

Articles will be included if they have been published from January 1998 to December 2020. This decision stems from the timeline of vaccine uptake and hesitancy. A published review of vaccine hesitancy discussed that, 'Since the late 1990s, concern has grown regarding a resurgence of the "anti-vaccine movement," a loosely defined group of individuals who sow doubt about the effectiveness and safety of vaccines'. ${ }^{23}$ The review noted that objections to vaccination have been present since the early 18th century, but 'the most current iteration of this scare can be traced to the publication of Andrew Wakefield's (since-retracted) paper linking the measlesmumps-rubella vaccine to autism in $1998{ }^{\prime} .^{23}$ Since this time frame has sparked much of the modern conversations surrounding vaccination and vaccine hesitancy, it will be used for the scoping review.

In terms of language, articles will be included if the full text is available in English, Arabic or French, as these are three of the most common languages in the Arab countries in the MENA region. Articles will be excluded if written in any language other than the abovementioned and for which open-access automated translation programmes such as Google Translate are not suitable. Articles that do not focus on the human vaccine and that do not involve populations in Arab countries in the MENA will be excluded. All types of evidence will be included, including research studies, review articles, grey literature and policy reports.

\section{Types of participants}

Target participants for this review include any individuals living in Arab countries in the MENA region based on the World Bank country list, which includes Algeria, Bahrain, Djibouti, Egypt, Iraq, Jordan, Kuwait, Lebanon, Libya, Morocco, Oman, Qatar, Saudi Arabia, Syria, Tunisia, the United Arabic Emirates, West Bank and Gaza, and Yemen. ${ }^{89}$

\section{Concept}

The main concept that is addressed by the scoping review is vaccine hesitancy. According to the Strategic Advisory Group of Experts on Immuniation (SAGE) Working Group on Vaccine Hesitancy, vaccine hesitancy is defined as the 'delay in acceptance or refusal of vaccination despite availability of vaccination services'. ${ }^{2}$ Vaccine hesitancy itself is a complex issue that varies across time and place, and for that reason, this concept will then be 


\section{Table 1 PICO model}

\begin{tabular}{|c|c|c|c|}
\hline Participant/population & Intervention & Comparators & Outcomes \\
\hline $\begin{array}{l}\text { Middle East, North Africa, Arab, } \\
\text { Muslim, MENA, Algeria, Bahrain, } \\
\text { Djibouti, Egypt, Iraq, Jordan, Kuwait, } \\
\text { Lebanon, Libya, Malta, Morocco, } \\
\text { Oman, Qatar, Saudi Arabia, Syria, } \\
\text { Tunisia, United Arabic Emirates, } \\
\text { West Bank and Gaza, Palestine and } \\
\text { Yemen }\end{array}$ & $\begin{array}{l}\text { Vaccination, vaccine, } \\
\text { immunisation, inoculation }\end{array}$ & $\begin{array}{l}\text { There will be no } \\
\text { designated comparators } \\
\text { for the model. }\end{array}$ & $\begin{array}{l}\text { Vaccine confidence, vaccine uptake, } \\
\text { vaccine refusal, vaccine hesitancy, } \\
\text { vaccine delay, missed schedule of } \\
\text { vaccine, non-medical vaccination } \\
\text { exemption }\end{array}$ \\
\hline
\end{tabular}

MENA, Middle East and North Africa; PICO, Participants-Intervention-Comparators-Outcome.

applied to the specific context of the study, as specified below to be Arab countries in the MENA region.

\section{Context}

The context of this review will include Arab countries in the MENA region, and thus the cultural and religious heritage of individuals in the countries (including but not limited to religion, language and health-seeking traditions) as well as the location/place where vaccination services are provided.

\section{Searching}

The primary search strategy was developed for PubMed and Embase, and similar searches will be executed in Cochrane, Scopus and any other databases as needed. The databases will be searched from 1 January 1998 to
31 December 2020. In addition, we hope to include grey literature available on the topic and will search Google and Google Scholar in addition to the aforementioned databases. We anticipate that the search for articles will be run across all databases in October and November 2020.

Studies will be selected according to elements of the Participants-Intervention-Comparators-Outcome model,${ }^{24}$ as outlined in table 1 . While the model includes clear participants, interventions and outcomes, there will be no designated comparator. Although a comparison is typically beneficial for evaluating the impact of the intervention, given the limited scope of literature on the topic, we have avoided restricting the literature to specific comparators and will instead widely capture literature that addresses vaccine hesitancy in the region.

Table 2 Search protocol

\section{Search category PubMed}

Intervention

(1) vaccin* $\left[\right.$ tw] OR immunis* $\left[\right.$ tw] OR immuniz ${ }^{*}[\mathrm{tw}]$ OR inocul* OR 'vaccination'[MeSH] OR 'immunization'[MeSH] OR 'vaccines'[MeSH] OR 'immunization programs' [MeSH]

Outcome

(2) 'vaccination refusal'[MeSH] OR (treatment refusal[MeSH] AND vaccination[MeSH]) OR 'anti-vaccination Movement' $[\mathrm{MeSH}] \mathrm{OR}$ accept*[tw] OR anxiet*[tw] OR anti-vacc*[tw] OR anti-vaxx*[tw] OR antivacc ${ }^{*}[\mathrm{tw}]$ OR antivaxx*[tw] OR attitude*[tw] OR autis*[tw] OR avoid*[tw] OR aware* [tw] OR barrier*[tw] OR behavior*[tw] OR behaviour ${ }^{*}[\mathrm{tw}]$ OR concern*[tw] OR confiden*[tw] OR compulsory[tw] OR compel*[tw]

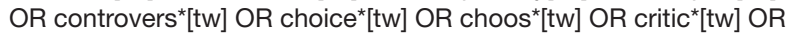
decid $^{*}[\mathrm{tw}]$ OR delay ${ }^{*}[\mathrm{tw}]$ OR deny ${ }^{*}[\mathrm{tw}]$ OR deni*$[\mathrm{tw}]$ OR decision* ${ }^{*}[\mathrm{tw}]$ OR dilemma*[tw] OR disinform*[tw] OR distrust*[tw] OR doubt*[tw] OR dropout[tw] OR exempt* $t^{\star}[\mathrm{tw}]$ OR fear ${ }^{*}[\mathrm{tw}]$ OR hesita* $[\mathrm{tw}]$ OR intent ${ }^{\star}[\mathrm{tw}]$ OR knowledge $^{*}[\mathrm{tw}]$ OR mandat ${ }^{*}[\mathrm{tw}]$ OR misconcept ${ }^{\star}[\mathrm{tw}]$ OR misinform ${ }^{*}[\mathrm{tw}]$ OR mistrust ${ }^{*}[\mathrm{tw}]$ OR MMR[tw] OR objection*[tw] OR objecting* ${ }^{*}[\mathrm{tw}]$ OR oppos*[tw] OR perception*[tw] OR perceiv*[tw] OR phobi*[tw] OR

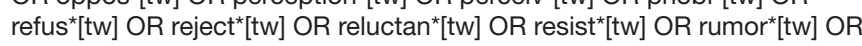
rumour ${ }^{*}[\mathrm{tw}]$ OR skeptic ${ }^{*}[\mathrm{tw}]$ OR trust* $[\mathrm{tw}]$ OR uptake ${ }^{*}[\mathrm{tw}]$

\begin{tabular}{|c|c|c|}
\hline Region & $\begin{array}{l}\text { (3) Middle East*[tw] OR Middle East[MeSH] OR North Africa*[tw] OR } \\
\text { Arab*[tw] OR Muslim*[tw] OR MENA[tw] OR Algeria*[tw] OR Bahrain*[tw] } \\
\text { OR Djibouti*[tw] OR Egypt*[tw] or Iraq }{ }^{*}[\text { tw] OR Jordan*[tw] OR Kuwait*[tw] } \\
\text { OR Leban*[tw] OR Jordan[tw] OR Libya*[tw] OR Morocc*[tw] OR } \\
\text { Oman*[tw] OR Qatar*[tw] OR Saudi Arabia*[tw] OR Saudi*[tw] OR } \\
\text { Syria*[tw] OR Tunisia*[tw] OR United Arab Emirates[tw] OR UAE[tw] } \\
\text { OR Emirat*[tw] OR West Bank[tw] OR Gaza[tw] OR Palestin*[tw] OR } \\
\text { Yemen*[tw] }\end{array}$ & 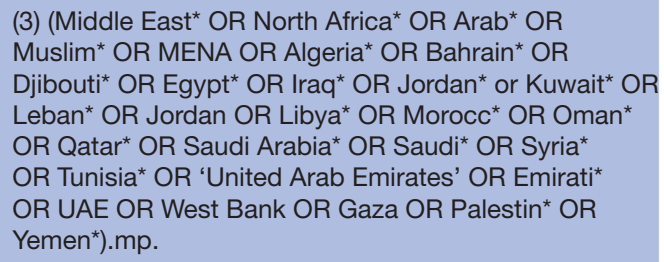 \\
\hline Date & (4) ‘1998/01/01’[PDat]: '2020/09/30’[PDat] & (4) $r d=19980101-20200930$ \\
\hline Results & 3870 & 3735 \\
\hline
\end{tabular}

\section{Embase}

(1) $\left(\right.$ vaccin $^{\star}$ OR immunis ${ }^{*}$ OR immuz ${ }^{*}$ OR inocul $\left.{ }^{\star}\right) \cdot m p$.

(2) (anti-vaccination movement OR vaccination refusal OR patient compliance OR accept* OR anxiet* OR

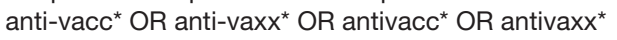
OR attitude ${ }^{\star}$ OR autis* OR avoid* OR aware* OR barrier $^{\star}$ OR behavior* OR behaviour ${ }^{\star}$ OR concern* OR confiden* OR compulsory OR compel ${ }^{*}$ OR controvers* OR choice* OR choos* OR critic* OR decid* OR delay* OR deny* OR deni* OR decision* OR dilemma* OR disinform* OR distrust* ${ }^{\star}$ OR doubt* OR dropout OR exempt ${ }^{\star}$ OR fear ${ }^{\star}$ OR hesita* OR intent ${ }^{\star}$ OR knowledge ${ }^{*}$ OR mandat* OR misconcept ${ }^{\star}$ OR misinform* OR

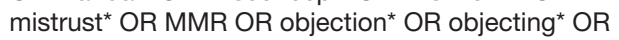
oppos* OR perception* OR perceiv* OR phobi* OR refus $^{*}$ or reject ${ }^{\star}$ or reluctan ${ }^{\star}$ OR resist ${ }^{\star}$ OR rumor ${ }^{\star}$ OR rumour $^{\star}$ OR skeptic ${ }^{\star}$ OR trust ${ }^{\star}$ OR uptake*).mp.

(3) (Middle East* OR North Africa* OR Arab* OR Muslim* OR MENA OR Algeria* OR Bahrain* OR Djibouti* OR Egypt* OR Iraq ${ }^{\star}$ OR Jordan* or Kuwait* OR OR Tunisia* OR 'United Arab Emirates' OR Emirati OR UAE OR West Bank OR Gaza OR Palestin* OR Yemen*).mp. Yemen* $[\mathrm{tw}]$

$\mathrm{rd}=19980101-20200930$

3735

MENA, Middle East and North Africa; MeSH, Medical Subject Headings; MMR, measles-mumps-rubella; UAE, United Arab Emirates. 
Additionally, to capture multiple dimensions of vaccine hesitancy in the MENA region, the search strategy will include the non-exhaustive list of keywords and Medical Subject Headings in table 2.

Once retrieved, all articles will first be screened by title and abstract by at least two reviewers to ascertain their relevance. When in doubt, the full article will be scanned to further determine its relevance or decide on its exclusion. Reference lists of relevant articles will also be perused to ensure literature saturation. Additionally, in order to determine inter-rater reliability, kappa will be reported for each pair of screeners in the review. This will be essential as a measure of how frequently the screeners were in agreement with one another. A kappa value of 0.80 or higher will be considered to indicate substantial reliability. Only when the first and second reviewers are unable to reach a consensus or decision for inclusion is not straightforward will a third reviewer be asked to provide input.

\section{Extracting and charting results}

'Data charting' in scoping reviews refers to the process of data extraction, the process by which the reader is provided with a clear and concise summary of results from articles relevant to the objective(s) of the review. ${ }^{22}$ For consistency, we have developed a data charting template for our review (table 3) to be used by all reviewers. This data charting template was created in consultation with the JBI Manual for Evidence Synthesis and Tankwanchi et $a .^{22}{ }^{25}$ It is possible that this form will change after a sample of studies has been charted by multiple reviewers.

\section{Summarising and reporting results}

The information provided by the data extraction from included studies will be collated and from there, quantitative results will be presented using descriptive statistics and qualitative results will be reported thematically. This will be followed by an informed discussion based on careful consideration of the results in keeping with the purpose and objective of the review. Through this, the goal is to provide a descriptive overview of currently available information on vaccine hesitancy in Arab countries in the MENA region. Additionally, however, if the data extraction from included studies indicates that many papers showed the circumstances surrounding vaccine hesitancy in these countries to vary from certain comparators, we can discuss comparators that appear in the selected papers.

\section{Dissemination and implementation}

We intend to use the findings in two main ways: first, to inform future research decisions and studies and, second, to incorporate the information obtained into actionable projects. We hope to conduct a systematic review in the future once we understand which countries and outcomes have been the subject of sufficient research that a systematic review is going to be warranted. Depending on the findings, we may also call for funding agencies,

Table 3 Data extraction

\section{Data charting table for scoping review of MENA vaccine hesitancy}

Citation details Author/s, date of publication, title, journal, volume, issue, pages

Type of evidence source Quantitative, qualitative, mixed methods; research, review, policy, perspective, ethnography, commentary, letter, unpublished report, media article, etc

\begin{tabular}{ll}
\hline Purpose & Objectives and questions posed in this study \\
\hline Population & Main characteristics of populations, communities or individuals in study \\
\hline Participants & Age, sex and number \\
\hline Country(ies) of study & Arab nation/s within MENA region of study \\
\hline Specific place of residence & Neighbourhood, village, city, town, camp, state or administrative region of participants \\
\hline Context & City, village, town, rural, camp, etc \\
\hline Religion & Religion/s of participants \\
\hline Native language & First language/s spoken by participants \\
\hline Ethnic/racial identity & Ethnic or racial identity of participants \\
\hline Location of & Neighbourhood, village, city, town, state, camp or administrative region of immunisation centre \\
immunisation centre & Outgroup members with whom participants are compared \\
\hline $\begin{array}{l}\text { Comparator } \\
\text { Concept }\end{array}$ & $\begin{array}{l}\text { Types of intervention attempted, evaluated or measured by study; duration of intervention and } \\
\text { measures }\end{array}$ \\
\hline Intervention & Effect of intervention on vaccination rate or perceptions of vaccination; measures \\
\hline $\begin{array}{l}\text { Outcome } \\
\text { Vaccine/s of focus }\end{array}$ & specific vaccine/s accepted, delayed, rejected \\
\hline
\end{tabular}

MENA, Middle East and North Africa. 
governments and researchers to working more in these areas where we have identified gaps. We also hope to identify if there are key papers that should be offered in Arabic so that policy makers who do not speak English can access them. It is not until the problem has been readily identified and been made available to the necessary parties that the unique causes of vaccine hesitancy in Arab countries in the MENA region can be worked towards.

\section{Patient and public involvement}

This review will be based solely on published articles and will not involve any patients or the public.

Acknowledgements The authors would like to thank Kate Nyhan and Ola El Zein for guidance in developing the search strategy.

Contributors All authors have reviewed the submitted manuscript and approved the manuscript for submission. BG led the development of the study and worked to prepare the manuscript. SL and SA-A contributed to the development of the study and to the preparation of the manuscript. Finally, KK worked with the other authors to develop the study methodology and to oversee its implementation.

Funding The authors have not declared a specific grant for this research from any funding agency in the public, commercial or not-for-profit sectors.

\section{Competing interests None declared.}

Patient and public involvement Patients and/or the public were not involved in the design, or conduct, or reporting, or dissemination plans of this research.

Patient consent for publication Not applicable.

Provenance and peer review Not commissioned; externally peer reviewed.

Open access This is an open access article distributed in accordance with the Creative Commons Attribution Non Commercial (CC BY-NC 4.0) license, which permits others to distribute, remix, adapt, build upon this work non-commercially, and license their derivative works on different terms, provided the original work is properly cited, appropriate credit is given, any changes made indicated, and the use is non-commercial. See: http://creativecommons.org/licenses/by-nc/4.0/.

ORCID iD

Bayan Galal http://orcid.org/0000-0002-8821-864X

\section{REFERENCES}

1 World Health Organization. Ten threats to global health in 2019, 2019. Available: https://www.who.int/news-room/feature-stories/tenthreats-to-global-health-in-2019

2 MacDonald NE, SAGE Working Group on Vaccine Hesitancy. Vaccine hesitancy: definition, scope and determinants. Vaccine 2015:33:4161-4.

3 Plotkin S. History of vaccination. Proc Natl Acad Sci U S A 2014;111:12283-7.

4 Smith DR. Herd immunity. Vet Clin North Am Food Anim Pract 2019;35:593-604.
5 Châtelet Pdu I, Antona D, Freymuth F. Spotlight on measles 2010: update on the ongoing measles outbreak in France, 2008-2010. Euro Surveill 2010;15.

6 De Serres G, Markowski F, Toth E, et al. Largest measles epidemic in North America in a decade--Quebec, Canada, 2011: contribution of susceptibility, serendipity, and superspreading events. J Infect Dis 2013;207:990-8.

7 Falagas ME, Zarkadoulia E. Factors associated with suboptimal compliance to vaccinations in children in developed countries: a systematic review. Curr Med Res Opin 2008;24:1719-41.

8 The World Bank. Arab world the world bank group. Available: https:// data.worldbank.org/country/1A

9 The World Bank. Middle East and North Africa: the world bank group. Available: https://www.worldbank.org/en/region/mena

10 Alsubaie SS, Gosadi IM, Alsaadi BM, et al. Vaccine hesitancy among Saudi parents and its determinants. result from the who SAGE Working group on vaccine hesitancy survey tool. Saudi Med J 2019;40:1242-50.

11 Hussain A, Ali S, Ahmed M, et al. The Anti-vaccination movement: a regression in modern medicine. Cureus 2018;10:e2919.

12 Memri. Rising Attacks On Polio Workers In Pakistan - Media Report: Rumors That The Vaccine 'Is A CIA Plot To Sterilize Muslim Children'; Article: 'Jews, Who Dream Of Ruling The World, Have Invented. Vaccines, Drugs, And Injections. To Weaken Muslims', 2014. Available: https://www.memri.org/reports/rising-attacks-polioworkers-pakistan-media-report-rumors-vaccine-cia-plot-sterilizemuslim

13 Zuzak TJ, Zuzak-Siegrist I, Rist L, Staubli G, et al. Attitudes towards vaccination: users of complementary and alternative medicine versus non-users. Swiss Med Wkly 2008;138:713-8.

14 Ahmed A, Lee KS, Bukhsh A, et al. Outbreak of vaccine-preventable diseases in Muslim majority countries. $J$ Infect Public Health 2018;11:153-5.

15 Vaccarella S, Bruni L, Seoud M. Burden of human papillomavirus infections and related diseases in the extended middle East and North Africa region. Vaccine 2013;31 Suppl 6:G32-44.

16 Abdulmalik Al. Spreading anti-vaccine rumors a threat to public health, 2019. Available: https://www.arabnews.com/node/1442796

17 Gamaoun R. Knowledge, awareness and acceptability of antiHPV vaccine in the Arab states of the middle East and North Africa region: a systematic review. East Mediterr Health $\mathrm{J}$ 2018;24:538-48.

18 Division NHaM. Virtual Meeting on Improving Vaccine Uptake - Issues of Access and Hesitancy. YouTube, 2020.

19 COCONEL Group. A future vaccination campaign against COVID-19 at risk of vaccine hesitancy and politicisation. Lancet Infect Dis 2020;20:769-70.

20 Peters MDJ, Godfrey CM, Khalil H, et al. Guidance for conducting systematic scoping reviews. Int J Evid Based Healthc 2015;13:141-6.

21 Larson HJ, de Figueiredo A, Xiahong Z, et al. The state of vaccine confidence 2016: global insights through a 67-Country survey. EBioMedicine 2016;12:295-301.

22 Aromataris EMZ. JBI manual for evidence synthesis. JBI, 2020.

23 Smith TC. Vaccine rejection and Hesitancy: a review and call to action. Open Forum Infect Dis 2017;4:ofx146.

24 Libraries N. Health (nursing, medicine, allied health): search strategies: framing the question (PICO). Available: https://guides.nyu. edu/c.php?g=276561\&p=1847897

25 Tankwanchi AS, Jaca A, Larson HJ, et al. Taking stock of vaccine hesitancy among migrants: a scoping review protocol. BMJ Open 2020;10:e035225. 